\title{
Israël : réformer les conditions d'emploi des enseignants pour améliorer l'apprentissage des élèves
}

Reut Gordon

\section{OpenEdition}

Journals

Édition électronique

URL : http://journals.openedition.org/ries/821

DOI : 10.4000/ries.821

ISSN : 2261-4265

Éditeur

Centre international d'études pédagogiques

\section{Édition imprimée}

Date de publication : 1 septembre 2010

Pagination : 14-17

ISBN : 978-2-85420-582-4

ISSN : $1254-4590$

Référence électronique

Reut Gordon, «Israël : réformer les conditions d'emploi des enseignants pour améliorer

l'apprentissage des élèves », Revue internationale d'éducation de Sèvres [En ligne], 54 | septembre 2010, mis en ligne le 01 septembre 2013, consulté le 02 mai 2019. URL : http://journals.openedition.org/ ries/821 ; DOI : 10.4000/ries.821

Ce document a été généré automatiquement le 2 mai 2019.

(c) Tous droits réservés 


\title{
Israël : réformer les conditions d'emploi des enseignants pour améliorer l'apprentissage des élèves
}

\author{
Reut Gordon
}

1 Un enfant qui pousse la porte de l'école avec des centaines d'autres élèves et s'assoit dans une salle de classe avec quarante autres enfants; une élève dont le professeur ne se souvient plus du nom, ne connaît pas les qualités, qu'il n'a pas entendu jouer de la musique ni vue réussir un dribble incroyable; une enseignante qui rentre chez elle frustrée parce qu'elle a manqué de temps, n'a pas réussi, a renoncé à ses rêves; un professeur dont le salaire, la première année d'enseignement, était inférieur au salaire minimum, qui percevait un "complément de revenu » et qui, au terme de sa carrière, après avoir acquis des diplômes d'études supérieures, continué à étudier et effectué des stages pendant ses années de service, finit par recevoir le salaire national moyen... En Israël, les élèves et les professeurs ont longtemps attendu la réforme qui améliorerait de manière tangible les conditions d'apprentissage des élèves ainsi que le statut des professeurs et leurs conditions d'emploi.

2 L'un des nombreux facteurs ayant entraîné des difficultés au niveau du système éducatif israélien ${ }^{1}$, au cours des années passées, est l'instabilité gouvernementale et donc les changements fréquents de ministre de l'Éducation - conséquence du système politique israélien et des équilibres de pouvoir entre la gauche et la droite pendant de nombreuses années. Chaque nouveau ministre changeait la politique de son prédécesseur et le système a souffert d'un manque de continuité, sans que pour autant les conditions d'apprentissage des élèves ou les conditions de travail des professeurs évoluent de façon significative. Jusqu'à présent, un poste d'enseignant en Israël comprenait essentiellement des heures d'enseignement effectives (trente heures hebdomadaires pour l'école élémentaire et vingt-quatre heures au-delà du primaire). Par conséquent, les élèves passaient plus de temps à l'école que les professeurs et ne rencontraient ces derniers que pendant les cours, dans des classes de quarante élèves (bien souvent). Un enfant qui 
quittait la salle de classe et se retrouvait dans le couloir, par exemple parce qu'il était malade ou en raison d'un problème de discipline, ne croisait généralement pas d'adulte, puisque les professeurs n'étaient pas dans l'établissement en dehors de leurs heures de cours.

Les objectifs éducatifs et organisationnels que la réforme s'efforce d'atteindre sont ainsi formulés :

- consolider le statut des professeurs et augmenter leur salaire ;

- réduire les écarts entre le centre et la périphérie ainsi qu'entre les différentes strates de la société ;

- donner à chaque élève une réelle égalité des chances ;

- améliorer la réussite des élèves ;

- renforcer l'éducation aux valeurs et à la citoyenneté dans le système éducatif ;

- améliorer le climat scolaire et lutter contre la violence à l'école.

4 L'innovation, dans la réforme "Nouvel Horizon ", ne tient pas tant aux objectifs fixés qu'à la création de mécanismes d'organisation garantissant sa pérennité malgré un climat d'instabilité politique. Il a donc été décidé d'inscrire la réforme dans le cadre d'accords salariaux, qui sont relativement plus stables que des décisions gouvernementales facilement modifiables. Le gouvernement a accordé une rallonge budgétaire à l'éducation pour instaurer la réforme, afin d'augmenter les salaires et de financer la mise en place d'enseignements en petits groupes. Les principes de la réforme ont été élaborés en collaboration avec le Syndicat des enseignants d'Israël qui représente les professeurs des écoles élémentaires, des jardins d'enfant et certains professeurs du secondaire inférieur. Cependant, l'Organisation des enseignants du secondaire, qui représente les enseignants du secondaire supérieur et une partie de ceux du secondaire inférieur, a critiqué la mise en place de cette réforme. L'Histadrut (Fédération des travailleurs israéliens) a soutenu le concept de la réforme, mais a stipulé qu'elle était favorable à une hausse conséquente des salaires des enseignants pour tout travail supplémentaire, afin que les changements ne soit pas perçus comme une rupture des accords salariaux.

Environ deux mois après la rentrée de l'année scolaire 2007-2008, quelque trois cents écoles ont été recrutées pour expérimenter la réforme "Nouvel horizon", avec la promesse faite à tous les enseignants participants de pouvoir retourner à l'ancien système s'ils le désiraient, à la fin de l'expérimentation. Il a été décidé de commencer l'expérimentation alors même que l'élaboration des nouveaux accords salariaux était en cours, afin de pouvoir prendre en compte les difficultés rencontrées et les opportunités offertes aux enseignants et aux chefs d'établissement, et donc d'élaborer les accords en même temps que les principes de la réforme étaient mis en place. Le principal changement a porté sur la position de l'enseignant: dans une école élémentaire, un professeur à plein temps effectuerait dorénavant trente-six heures, soit vingt-six heures d'enseignement en classe entière, cinq heures dévolues au travail en petits groupes de un à cinq élèves et cinq heures de présence dans l'établissement pour participer à d'autres activités.

6 Pour les élèves, le principal changement a consisté dans le volume horaire désormais consacré aux cours à effectifs réduits permettant un enseignement personnalisé et de qualité. Deux des cinq heures de chaque professeur en effectifs réduits devaient servir à l'instauration de discussions personnelles avec les élèves et les trois heures restantes au renforcement de l'enseignement des matières fondamentales du curriculum - (langue 
maternelle, mathématiques, sciences et anglais), pour les élèves les plus faibles comme pour les meilleurs.

7 Pour les professeurs, le principal changement a consisté en l'allongement de la semaine de travail et la nécessité d'enrichir leurs pratiques pédagogiques pour s'adapter aux spécificités du travail en petit groupe. On s'est aperçu que les professeurs avaient besoin de nouveaux outils pédagogiques pour mener les rencontres individuelles avec les élèves et pour faire face aux informations problématiques (abordées parfois au cours des conversations) concernant la vie des enfants à la maison ou avec d'autres enfants. En outre, beaucoup d'enseignants avaient recours aux méthodes d'enseignement auxquelles ils étaient habitués (méthodes adaptées aux classes entières surchargées) pour les cours en effectif réduit et restaient dans une dynamique frontale d'enseignement, avec le modèle d'enseignement "en soleil », où le professeur interroge et les élèves répondent. Les professeurs n'ont vraiment été en mesure d'introduire de nouvelles pratiques pédagogiques que lorsqu'ils ont assimilé les changements et se sont habitués au nouvel emploi du temps. Ils ont commencé alors à considérer les groupes d'apprentissage comme des groupes de pairs fonctionnant comme des matrices, dans lesquelles le rôle du professeur est de faciliter, de guider et de donner des instructions.

Le modèle « en soleil »

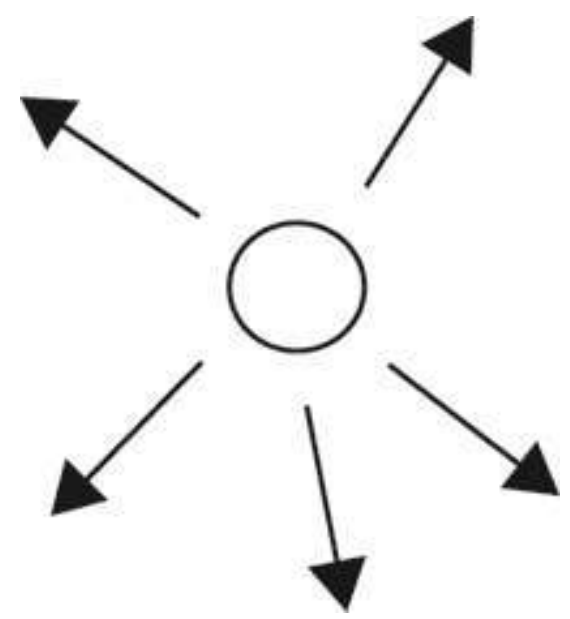




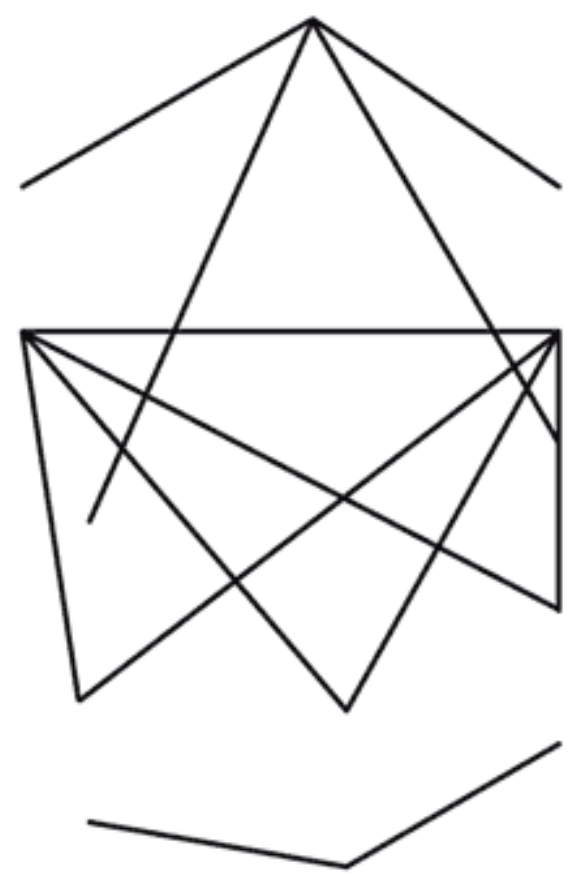

8 Une unité spéciale a été mise en place au ministère de l'Éducation pour planifier la formation continue des enseignants: d'une part, les besoins du système et les sujets d'études ont été précisés, et d'autre part, les niveaux de salaires corrélés à la formation continue ont été définis de sorte que, pour la première fois, les professeurs ont pu voir leur salaire augmenter non seulement du fait de leur ancienneté, mais aussi du fait de leur montée en grade.

9 Au début, les professeurs des premières écoles qui ont testé la réforme Nouvel Horizon se déclaraient totalement surmenés. Ce constat servait d'argument aux enseignants d'autres établissements qui souhaitaient maintenir le status quo. Une opposition active et organisée de l'Organisation des enseignants du secondaire s'est manifestée, afin de persuader les enseignants de l'école élémentaire et aussi (et surtout) ceux du secondaire inférieur de ne pas rejoindre la réforme. Lors de la deuxième année d'expérimentation, l'une des conditions pour qu'un établissement décide de la mettre en place était l'accord de la majorité des enseignants. Dans de nombreux établissements, la plupart des quelques enseignants ayant refusé d'appliquer la réforme en début d'année ont finalement demandé à s'y rallier lorsqu'ils se sont rendu compte que les salaires de leurs collègues avaient été fortement revalorisés (d'environ $30 \%$ ) au cours des premiers mois, et que les heures d'enseignement personnalisé permettaient une véritable évolution de la relation avec les élèves et avaient un réel impact sur leur résultats. Au cours des années suivantes, lorsque les enseignants se sont habitués à travailler dans le cadre de la réforme et ont acquis l'expérience du travail en petits groupes, ils ont affirmé qu'ils étaient moins surmenés et qu'ils avaient le sentiment d'avoir atteint des objectifs professionnels perçus comme inaccessibles par le passé. 
Données sur l'application de la réforme pour l'année scolaire 2009-2010

\begin{tabular}{|c|c|c|c|c|c|}
\hline \multicolumn{4}{|c|}{ Enseignement classique } & \multicolumn{2}{|c|}{$\begin{array}{l}\text { Enseignement } \\
\text { spécialisé }\end{array}$} \\
\hline $\begin{array}{l}\text { Écoles } \\
\text { élémentaires }\end{array}$ & $\begin{array}{l}\text { Établissements } \\
\text { indépendants du } \\
\text { secondaire } \\
\text { inférieur }\end{array}$ & $\begin{array}{l}\text { Établissements du } \\
\text { secondaire inférieur } \\
\text { intégrés à des } \\
\text { établissements couvrant } \\
\text { tout le secondaire }\end{array}$ & $\begin{array}{l}\text { Jardins } \\
\text { d'enfants }\end{array}$ & Écoles & $\begin{array}{l}\text { Jardins } \\
\text { d'enfants }\end{array}$ \\
\hline $72,6 \%$ & $44,07 \%$ & $5,25 \%$ & $4,92 \%$ & $17,59 \%$ & $2 \%$ \\
\hline
\end{tabular}

10 Un nouveau changement de gouvernement est survenu après la deuxième année de réforme (2009). Lors de la prise de fonction du nouveau ministre de l'Éducation, 813 écoles élémentaires et 50 établissements de la première moitié du secondaire étaient passés à la réforme. À la fin de sa première année d'exercice, 1138 écoles élémentaires et 101 établissements du secondaire inférieur avaient adopté ce nouveau mode de fonctionnement. Au début de la troisième année, il a été décidé que la réforme serait désormais une politique du ministère de l'Éducation et ne dépendrait plus de l'accord de la majorité des enseignants. Cela a provoqué un conflit avec l'Organisation des enseignants du secondaire mais le ministère de l'Éducation l'a emporté.

11 Bien que le système éducatif soit encore en phase d'apprentissage et d'élaboration de la réforme, on peut déjà s'accorder à dire qu'il est manifeste que le dialogue personnel entre les professeurs et les élèves améliore le climat scolaire, le ressenti des élèves et des professeurs et, comme l'on pouvait s'y attendre, que l'enseignement personnalisé permet un meilleur apprentissage et une plus grande réussite.

Dans une école de Safed, en Galilée, j'ai rencontré des élèves de 11 ans qui rayonnaient de joie en cours de soutien d'anglais. Ils m'ont affirmé qu'avant la réforme, ils avaient honte lorsqu'ils participaient en cours, détestaient la matière et essayaient d'être "malades » les jours de cours d'anglais. Depuis la réforme, ils apprennent en petits groupes au sein desquels, selon eux, ils éprouvent moins de gêne, acquièrent plus de connaissances et s'entraînent à prononcer correctement à haute voix. Aujourd'hui, c'est leur matière préférée et leur seule hantise est que les cours en effectifs réduits s'arrêtent, puisqu'ils sont maintenant au niveau du reste de la classe.

\section{NOTES DE FIN}

1. . Il existe en Israël trois secteurs publics subventionnés par l'État intégralement et un quatrième « reconnu mais non officiel» partiellement pris en charge. Voir D. Charbit et M. Darmoni (2004), « Religion(s) et école(s) en Israël », RIE n³6, p. 85-99. 
INDEX

Mots-clés : enseignant, réforme de l'enseignement

Index géographique : Israël

\section{AUTEUR}

\section{REUT GORDON}

Reut Gordon a été principale de deux écoles élémentaires, a travaillé comme adjointe du responsable de la réforme " Nouvel Horizon » au ministère de l'Éducation. Aujourd'hui, elle est doctorante en sciences de l'éducation à l'Université de Tel-Aviv et travaille au ministère, au Bureau des élèves doués et surdoués. 